01

\title{
Влияние термических сопротивлений на холодильный коэффициент термоэлектрической системы охлаждения
}

\author{
(C) E.H. Васильев
}

Институт вычислительного моделирования СО РАН, 660036 Красноярск, Россия

e-mail: ven@icm.krasn.ru

Поступило в Редакцию 22 октября 2020 г.

В окончательной редакции 8 декабря 2020 r.

Принято к публикации 9 декабря 2020 г.

Рассмотрена термоэлектрическая система охлаждения и терморегулирования, состоящая из термоэлектрического модуля, устройств подвода и отвода теплоты. На основе математической модели, использующей в качестве исходных данных рабочие характеристики серийного термоэлектрического модуля, проведены расчеты холодильного коэффициента системы охлаждения с учетом термических сопротивлений устройств подвода и отвода теплоты. Получены зависимости холодильного коэффициента от силы тока при различных значениях параметров охлаждения. Проведен анализ оптимальных значений силы тока питания термоэлектрического модуля.

Ключевые слова: термоэлектрический модуль, холодопроизводительность, термическое сопротивление.

DOI: 10.21883/JTF.2021.05.50684.296-20

\section{Введение}

В настоящее время термоэлектрические системы охлаждения и терморегулирования (ТЭСОТ) активно используются для охлаждения как микрообъектов, так и холодильных камер большого объема [1-3]. ТЭСОТ сохраняют работоспособность в любом пространственном положении, имеют высокую надежность и ресурс, возможность плавного и точного регулирования температурного режима вплоть до изменения режима охлаждения на режим нагрева. Главным недостатком ТЭСОТ является сравнительно низкий холодильный коэффициент $[4,5]$. Повышение эффективности ТЭСОТ может быть обеспечено как путем получения новых термоэлектрических материалов с улучшенными показателями добротности [6,7], так и за счет оптимизации конструкции и режимов работы системы, что позволяет максимально использовать потенциал существующих термоэлектрических материалов и устройств [8-10].

Термоэлектрический модуль (ТЭМ) по своей сути является тепловым насосом, производящим отрицательный температурный перепад за счет передачи теплоты с холодной стороны на горячую. Эффективность работы ТЭМ в значительной степени определяется потерями температурного перепада вследствие обратной теплопередачи от горячего спая к холодному за счет теплопроводности и джоулева тепловыделения. Величина таких потерь определяется добротностью термоэлектрического материала и режимом работы ТЭМ. На эффективность работы ТЭСОТ в целом дополнительное влияние оказывают потери температурного перепада в устройствах теплоподвода и теплоотвода, величина таких потерь зависит от термических сопротивлений этих устройств. В настоящей работе иссле- дуется влияние термических сопротивлений устройств подвода и отвода теплоты на холодильный коэффициент ТЭСОТ, анализ проведен на примере серийного модуля „S-127-14-11“, имеющего максимальные значения перепада температуры $\Delta T_{\mathrm{TEM}}=72.5^{\circ} \mathrm{C}$ и холодопроизводительности $Q=79.3 \mathrm{~W}$ при максимальной силе тока $I_{\max }=7.9 \mathrm{~A}[11]$.

\section{1. Тепловые процессы и холодильный коэффициент термоэлектрической системы охлаждения}

Принципиальная схема ТЭСОТ приведена на рис. 1. Тепловая мощность $Q$, выделяемая теплонагруженным элементом 1 , передается устройством теплоподвода 2 на холодную сторону ТЭМ 3. Теплопередающая способность устройства теплоподвода интегрально характери-

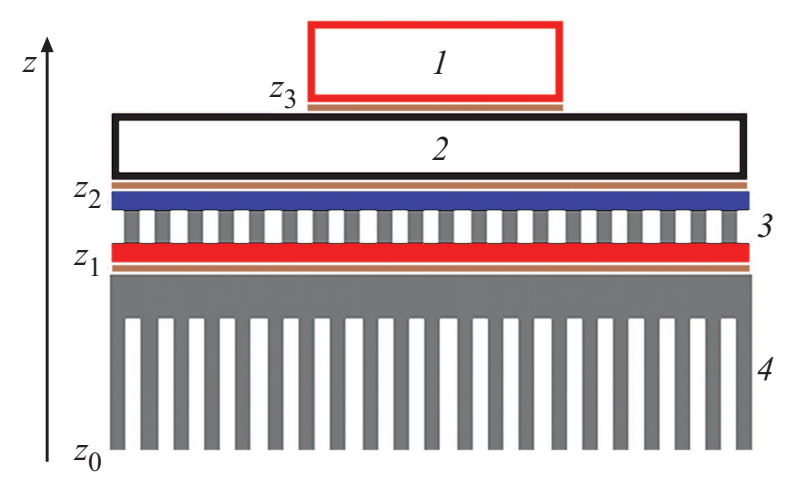

Рис. 1. Схема термоэлектрической системы охлаждения и терморегулирования: 1 - теплонагруженный элемент; 2 - устройство теплоподвода; 3 - ТЭМ; 4 - устройство теплоотвода. 


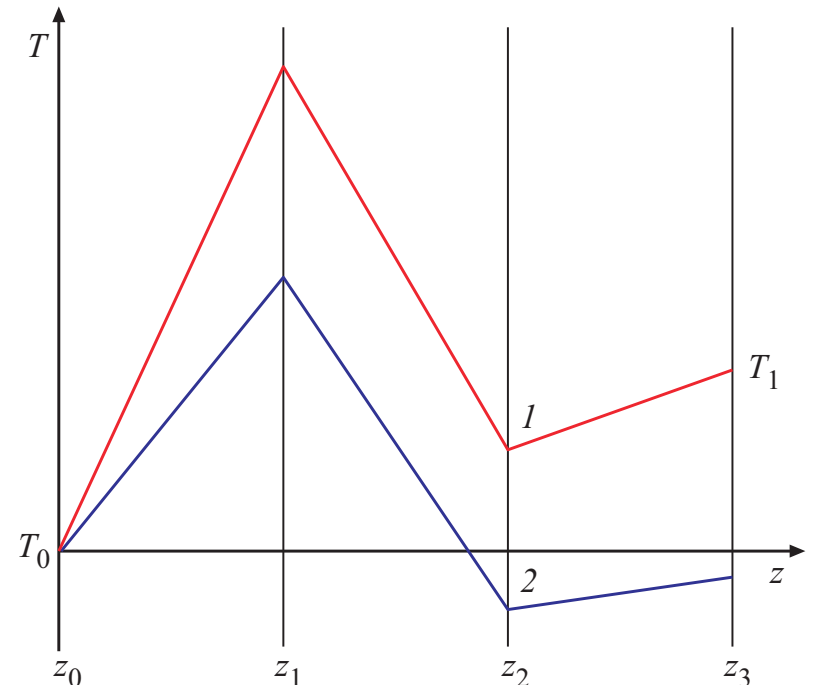

Рис. 2. Распределения температуры в термоэлектрической системе охлаждения при двух разных соотношениях температурных перепадов.

зуется величиной термического сопротивления $R_{T}$, peзультаты расчетов и анализ значений $R_{T}$ для устройства теплоподвода в виде теплораспределяющей пластины приведены в [12]. ТЭМ при своей работе выделяет теплоту, равную мощности собственного энергопотребления $W$. Тепловая мощность $Q+W$ отводится с горячей стороны ТЭМ во внешнюю среду устройством теплоотвода 4, имеющим суммарное термическое сопротивление $R_{S}$. Информация по устройствам теплоотвода и характерным значениям $R_{S}$ приведена в [10]. По площади контакта $z_{0}$ устройством теплоотвода теплота передается во внешнюю среду, имеющую температуру $T_{0}$. Горячей и холодной сторонам ТЭМ соответствуют контактные границы $z_{1}$ и $z_{2}$. Нижняя поверхность основания теплонагруженного элемента (поверхность $z_{3}$ ) характеризуется средним значением температуры $T_{1}$.

Характерные варианты температурных распределений в направлении $z$, которые могут реализоваться в ТЭСОТ, приведены на рис. 2. Температура внешней среды $T_{0}$ является исходным значением, напрямую влияющим на температуру посадочного места теплонагруженного элемента. На термическом сопротивлении $R_{S}$ устройства теплоотвода устанавливается положительный температурный перепад, равный $R_{S}(Q+W)$. ТЭМ производит отрицательный температурный перепад $-\Delta T_{\mathrm{TEM}}$. Устройство теплоподвода с термическим сопротивлением $R_{T}$ обеспечивает положительный температурный перепад $R_{T} Q$. В зависимости от соотношения величины температурных перепадов общий перепад $\Delta T_{e}=T_{1}-T_{0}$, как показано на рис. 2, может иметь как положительное (линия 1 ), так и отрицательное значение (линия 2).

Энергетическая эффективность работы ТЭМ описывается известной характеристикой COP (coefficient of performance), равной отношению холодопроизводи- тельности ТЭМ к потребляемой им электрической мощности. Зависимости величины СОР от силы тока и температурного перепада серийных ТЭМ, как правило, предоставляются производителями. Холодильный коэффициент ТЭСОТ определяется аналогичным образом

$$
\varepsilon=\frac{Q}{W}
$$

Характеристика СОР соответствует идеализированному случаю, когда процессы теплоподвода к холодной стороне и теплоотвода от горячей стороны ТЭМ полагаются идеальными. В отсутствие потерь температурного перепада справедливо соотношение $\Delta T_{e}=-\Delta T_{\mathrm{TEM}}$. Традиционно для ТЭМ указывается значение температурного перепада, равное разности температур между горячей и холодной сторонами модуля, следовательно, $\Delta T_{\mathrm{TEM}}$ определено в противоположном направлении по сравнению с $\Delta T_{e}$ и поэтому имеет отрицательный знак.

На величину холодильного коэффициента реальной системы охлаждения, включающей в себя устройства подвода и отвода, оказывают влияние потери температурного перепада на термических сопротивлениях. Поэтому величина $\varepsilon$ у ТЭСОТ будет ниже, чем значение СОР у отдельного ТЭМ при равных перепадах температуры на их границах. Влияние потерь температурного перепада на величину $\varepsilon$ происходит неявным образом. Для обеспечения заданной величины $\Delta T_{e}$ в ТЭСОТ потери температурного перепада могут быть скомпенсированы только соответствующим увеличением $\Delta T_{\mathrm{TEM}}$, которое достигается за счет повышения энергопотребления $W$ и уменьшения холодопроизводительности. В итоге увеличение $\Delta T_{\mathrm{TEM}}$ приводит к снижению СОР модуля, что подтверждается зависимостью $\mathrm{COP}\left(\Delta T_{\mathrm{TEM}}\right)$, и холодильного коэффициента ТЭСОТ в целом.

Расчет величины холодильного коэффициента ТЭСОТ проводился с учетом рабочих характеристик ТЭМ с помощью соотношения, связывающего общий температурный перепад $\Delta T_{e}=T_{1}-T_{0}$ с перепадами температур на отдельных элементах ТЭСОТ

$$
\begin{aligned}
\Delta T_{e}= & R_{T} Q+R_{S}(Q+W)-\Delta T_{\mathrm{TEM}}=\left(R_{S}+R_{T}\right) Q \\
& +R_{S} U(I, Q) I-\Delta T_{\mathrm{TEM}}(I, Q)
\end{aligned}
$$

Здесь $\Delta T_{\mathrm{TEM}}$ - перепад температуры между горячей и холодной сторонами ТЭМ, $U, I-$ напряжение и сила тока электропитания ТЭМ. Рабочие характеристики термоэлектрического модуля $Q\left(\Delta T_{\mathrm{TEM}}\right)$ и $U\left(\Delta T_{\mathrm{TEM}}\right)$ являются исходными данными для определения зависимостей $U(I, Q)$ и $\Delta T_{\mathrm{TEM}}(I, Q)$ с помощью интерполяционных многочленов, методика расчета этих зависимостей приведена в [13]. Решение нелинейной алгебраической системы уравнений для заданных значений $R_{S}, R_{T}, \Delta T_{e}$ и $I$ позволяет рассчитать величины $Q, W$ и $\varepsilon$. 


\section{2. Обсуждение результатов}

Максимальное значение холодильного коэффициента ТЭСОТ достигается при отсутствии потерь температурного перепада $\left(R_{S}=R_{T}=0\right)$, в этом идеальном случае величина холодильного коэффициента равна СОР. Зависимости $\varepsilon(I)$ на рис. 3 отображены сплошными линиями для значений $\Delta T_{\text {TEM }}=0.1,0.25,0.5$ и $0.75 \Delta T_{\max }$, штриховыми линиями для тех значений $\Delta T_{\mathrm{TEM}}$ отображены графики СОР производителя ТЭМ [11]. Соответствие результатов расчета с данными производителя свидетельствует об адекватности вычислительного алгоритма.

Учет потерь температурного перепада на термических сопротивлениях неизбежно приводит к снижению холодильного коэффициента. Для определения влияния термического сопротивления устройства теплоотвода рас-

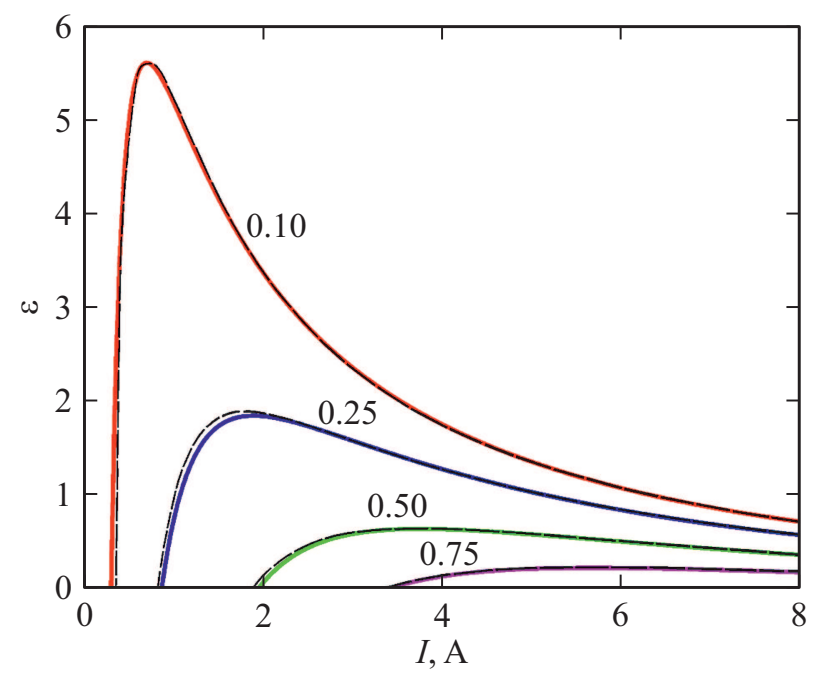

Рис. 3. Зависимости холодильного коэффициента от силы тока при значениях $\Delta T_{\text {TEM }}=0.1,0.25,0.5$ и $0.75 \Delta T_{\max }$.

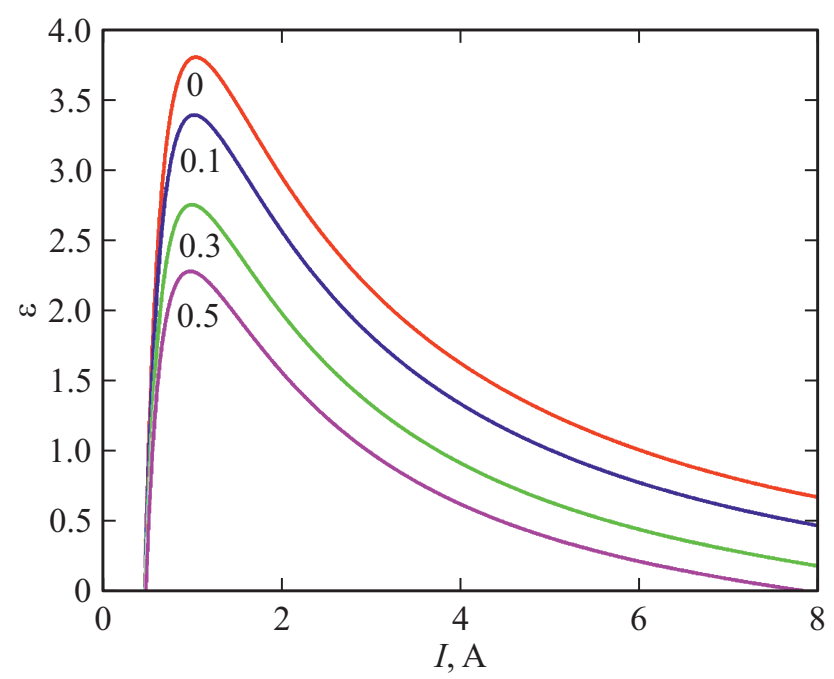

Рис. 4. Зависимости холодильного коэффициента от силы тока при $\Delta T_{e}=-10^{\circ} \mathrm{C}$. Числа у кривых - значения сопротивления $R_{S}$.

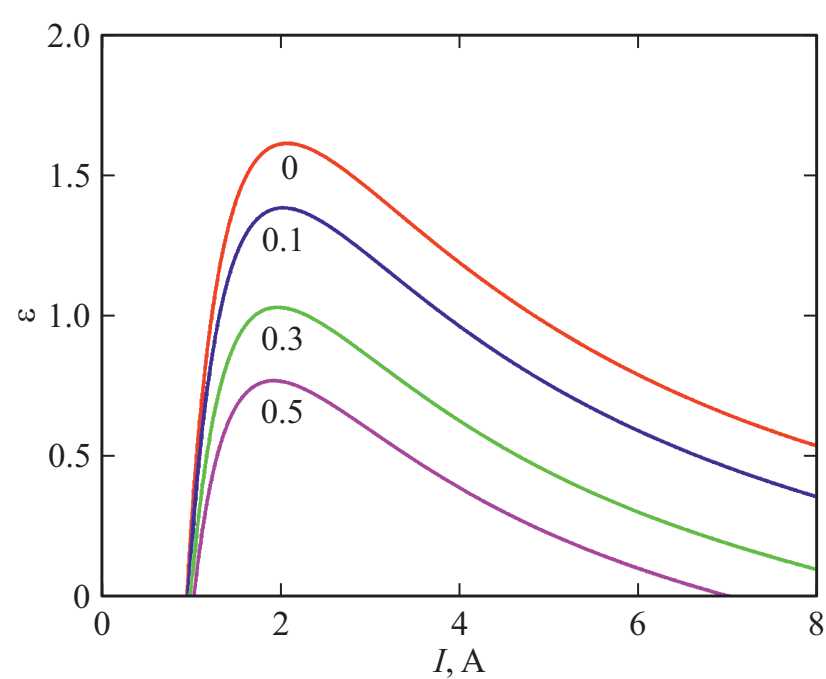

Рис. 5. Зависимости холодильного коэффициента от силы тока при $\Delta T_{e}=-20^{\circ} \mathrm{C}$. Числа у кривых - значения сопротивления $R_{S}$.

считаны зависимости $\varepsilon(I)$ без учета системы теплоподвода $\left(R_{T}=0\right)$ при значениях $R_{S}=0,0.1,0.3$ и $0.5 \mathrm{~K} / \mathrm{W}$. Для поддержания стабильной температуры теплонагруженного элемента система охлаждения должна работать в режиме терморегулирования, обеспечивая заданный температурный перепад $\Delta T_{e}$ между его основанием и внешней средой. На рис. 4 и 5 приведены зависимости $\varepsilon(I)$ для значений $\Delta T_{e}=-10$ и $-20^{\circ} \mathrm{C}$, величины $R_{S}$ указаны на соответствующих кривых. Из сопоставления зависимостей видно, что увеличение значения $R_{S}$ с 0 до 0.5 существенно снижает максимальное значение холодильного коэффициента: с 3.8 до 2.3 при $\Delta T_{e}=-10^{\circ} \mathrm{C}$ и с 1.6 до 0.77 при $\Delta T_{e}=-20^{\circ} \mathrm{C}$. При этом положение максимума зависимостей $\varepsilon(I)$ незначительно смещается в сторону уменьшения силы тока соответственно с 1 до $0.94 \mathrm{~A}$ для $\Delta T_{e}=-10^{\circ} \mathrm{C}$ и с 2.08 до $1.93 \mathrm{~A}$ для $\Delta T_{e}=-20^{\circ} \mathrm{C}$. Сравнение рис. 4 и 5 свидетельствует о значительном влиянии величины $\Delta T_{e}$ на холодильный коэффициент, при снижении величины $\Delta T_{e}$ на $10^{\circ} \mathrm{C}$ максимальные значения $\varepsilon$ упали примерно в 2.4 и 3 раза для $R_{S}=0$ и $0.5 \mathrm{~K} / \mathrm{W}$ соответственно.

Для определения влияния термического сопротивления устройства теплоподвода сначала рассмотрим зависимости холодильного коэффициента от силы тока, полученные при $\Delta T_{e}=-10^{\circ} \mathrm{C}$ и значениях $R_{T}=0,0.1$, 0.3 и $0.5 \mathrm{~K} / \mathrm{W}$ без учета системы теплоотвода $\left(R_{S}=0\right)$. На рис. 6 приведены зависимости $\varepsilon(I)$, на которых указаны соответствующие значения $R_{T}$. Сравнение зависимостей на рис. 4 и 6 показывает, что величина $R_{T}$ оказывает на холодильный коэффициент меньшее влияние, чем $R_{S}$. Отличие наиболее существенно при больших значениях силы тока, так, при $I=6 \mathrm{~A} \approx 0.75 I_{\max }$ изменение $R_{T}$ с 0 до $0.5 \mathrm{~K} / \mathrm{W}$ приводит к уменьшению $\varepsilon$ с 1 до 0.6 (рис. 6), а при аналогичном изменении $R_{S}$ величина $\varepsilon$ снижается с 1 до 0.2 (рис. 4). Такая разница обусловлена со- 


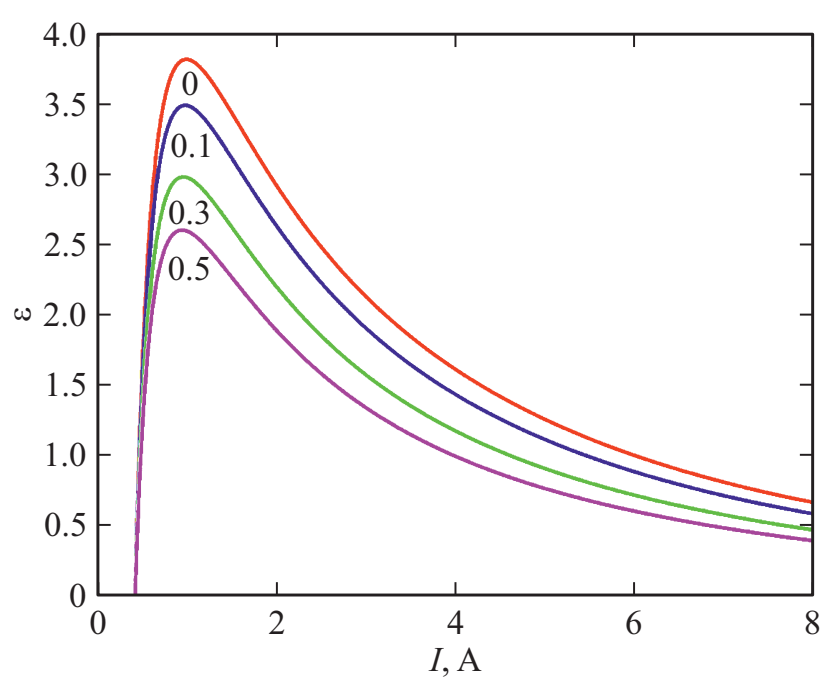

Рис. 6. Зависимости холодильного коэффициента от силы тока при значении $R_{S}=0$. Числа у кривых - значения сопротивления $R_{T}$.

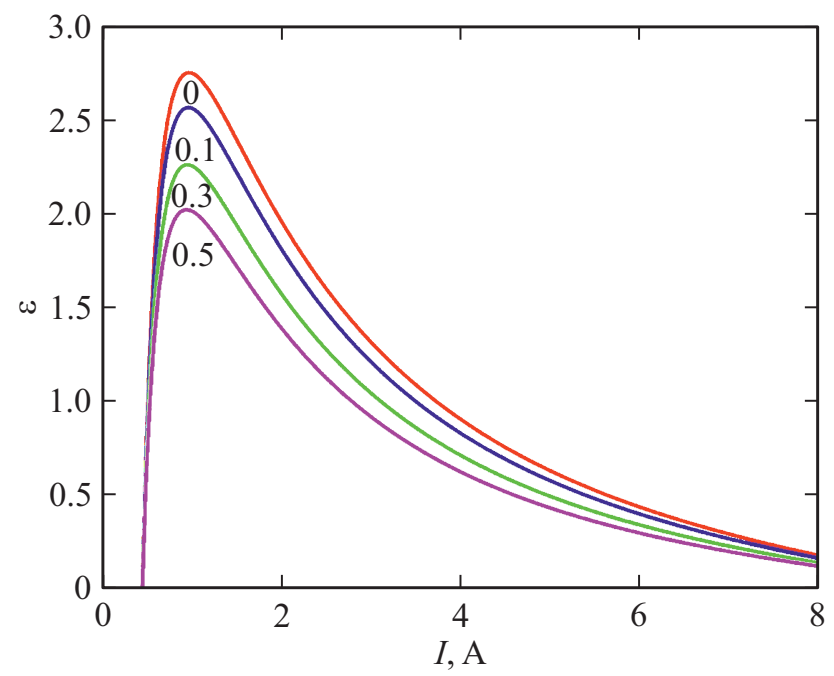

Рис. 7. Зависимости холодильного коэффициента от силы тока при значении $R_{S}=0.3 \mathrm{~K} / \mathrm{W}$. Числа у кривых - значения сопротивления $R_{T}$.

отношением потерь температурного перепада, которые пропорциональны тепловым мощностям $Q$ и $Q+W$ на устройствах теплоподвода и теплоотвода соответственно. Совместное влияние термических сопротивлений можно оценить по расчетным результатам, приведенным на рис. 7. Зависимости получены при $\Delta T_{e}=-10^{\circ} \mathrm{C}$, $R_{S}=0.3 \mathrm{~K} / \mathrm{W}$ и $R_{T}=0,0.1,0.3$ и $0.5 \mathrm{~K} / \mathrm{W}$, значения $R_{T}$ указаны на соответствующих кривых.

При выборе режима работы ТЭСОТ, помимо учета величины холодильного коэффициента, характеризующего экономичность процесса, также необходимо обеспечить холодопроизводительность, равную мощности тепловыделения теплонагруженного элемента при заданном температурном перепаде $\Delta T_{e}$ между его основанием и внешней средой. На рис. 8 приведены зависимости от силы тока величин, определяющих холодильный коэффициент. Жирными линиями отображены графики холодопроизводительности $Q(I)$, тонкими линиями зависимости мощности энергопотребления $W(I)$. Кривые 1 соответствуют идеальному случаю $R_{S}=R_{T}=0$, при расчете зависимостей 2 задавалось термическое сопротивление теплоотвода $R_{S}=0.3 \mathrm{~K} / \mathrm{W}$, при определении 3 учитывались оба термических сопротивления $R_{S}=R_{T}=0.3 \mathrm{~K} / \mathrm{W}$. Как видно из рис. 8 , влияние термических сопротивлений приводит к существенному снижению холодопроизводительности, так, при $I=6$ А величина $Q$ снизилась более чем в 2 раза по сравнению с идеальным случаем. Снижение холодопроизводительности ТЭСОТ обусловлено потерями температурного перепада на термических сопротивлениях, для обеспечения заданной величины $\Delta T_{e}$ эти потери компенсируются соответствующим увеличением $\Delta T_{\mathrm{TEM}}$, что, согласно нагрузочным прямым, приводит к снижению $Q$. На рис. 9 приведены зависимости $\Delta T_{e}$ для тех же значений термических сопротивлений: $1-R_{S}=R_{T}=0$; $2-R_{S}=0.3 \mathrm{~K} / \mathrm{W}, R_{T}=0 ; 3-R_{S}=R_{T}=0.3 \mathrm{~K} / \mathrm{W}$. Для заданного значения силы тока величина потерь температурного перепада, которую необходимо компенсировать, определяется по разнице значений $\Delta T_{e}$ на графиках 2 или 3 относительно идеального случая 1 .

Актуальной задачей, определяющей режим охлаждения ТЭСОТ, является выбор величины тока питания ТЭМ. Производители термоэлектрических систем в сопроводительной документации обычно предлагают в качестве оптимального параметра электропитания ТЭМ уровень 0.75 от максимальных значений тока или напряжения [14,15]. Рассчитанные зависимости $\varepsilon(I)$ и $Q(I)$ дают возможность определить оптимальные значения силы тока с учетом термических сопротивлений. Как видно на рис. 8, зависимости $Q(I)$ (кривые 2 и 3 ) имеют

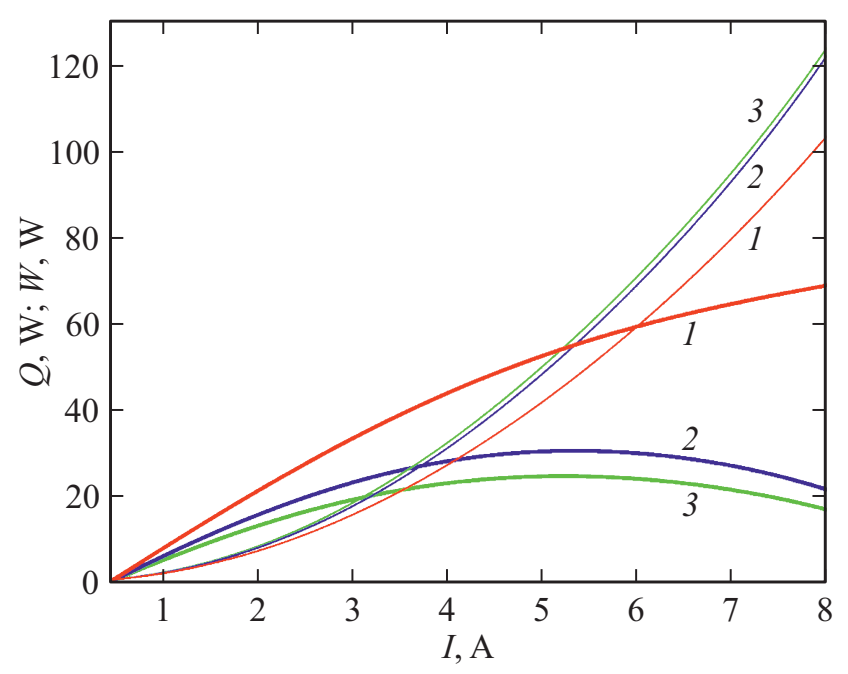

Рис. 8. Зависимости $Q$ (жирные линии) и $W$ (тонкие линии) от силы тока: $1-R_{S}=0, R_{T}=0 ; 2-R_{S}=0.3, R_{T}=0 ; 3-$ $R_{S}=0.3 \mathrm{~K} / \mathrm{W}, R_{T}=0.3 \mathrm{~K} / \mathrm{W}$. 


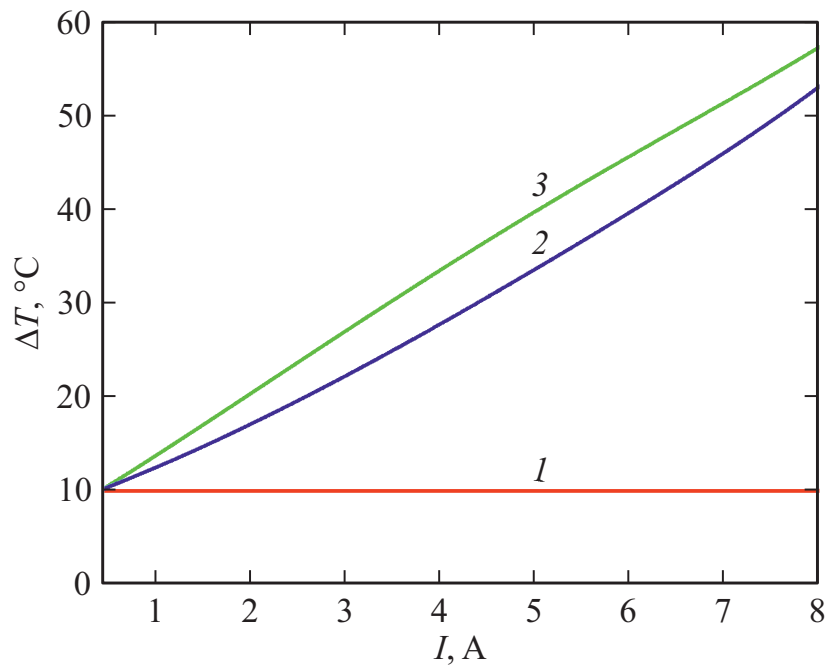

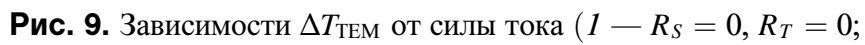
$\left.2-R_{S}=0.3 \mathrm{~K} / \mathrm{W}, R_{T}=0 ; 3-R_{S}=0.3 \mathrm{~K} / \mathrm{W}, R_{T}=0.3 \mathrm{~K} / \mathrm{W}\right)$.

максимум $Q^{*}$ при значениях $I_{Q}=5.2$ и $5.3 \mathrm{~A}$. Следовательно, рекомендуемый производителями уровень $0.75 I_{\max } \approx 6 \mathrm{~A}$ при учете термических сопротивлений не является оптимальным по величине $Q$, и еще в большей степени по величине $\varepsilon$. Значение $I_{Q}$ задает правую границу диапазона рабочих токов, соответствующую режиму максимальной холодопроизводительности ТЭСОТ. С учетом пологого характера $Q(I)$ целесообразнее выбирать меньшее значение $I$, например, при токе $I=3.7 \mathrm{~A}$, определенном по уровню $0.9 Q^{*}$ (для кривой 3 , рис. 8), значение холодильного коэффициента возрастет на $73 \%$ (с 0.45 при $I=5.2$ А до 0.78 ). Левую границу диапазона рабочих токов устанавливает значение $I_{\varepsilon}$, обеспечивающее максимальный холодильный коэффициент $\varepsilon^{*}$; для значений $R_{S}=R_{T}=0.3 \mathrm{~K} / \mathrm{W}$ величина $I_{\varepsilon}=0.94 \mathrm{~A}$ (рис. 7). Аналогично увеличение силы тока до значения $I=1.37 \mathrm{~A}$, определенного по уровню $0.9 \varepsilon^{*}$, дает рост холопроизводительности на $78 \%$ (с 4.6 до $8.2 \mathrm{~W}$ ). Таким образом, для рассматриваемых параметров ТЭСОТ (ТЭМ „S-127-14-11“, $\left.\Delta T_{e}=-10^{\circ} \mathrm{C}, R_{S}=R_{T}=0.3 \mathrm{~K} / \mathrm{W}\right)$ диапазон значений силы тока $1.37-3.7$ А близок к оптимальному, конкретное значение силы тока определяется с учетом мощности тепловыделения теплонагруженного элемента.

\section{Заключение}

Результаты расчетов на примере серийного термоэлектрического модуля „,S-127-14-11“ показали значительное влияние термических сопротивлений на характеристики термоэлектрической системы охлаждения. Потери температурного перепада на термических сопротивлениях устройств подвода и отвода теплоты приводят к росту разницы температур на ТЭМ, что обусловливает снижение холодильного коэффициента и холодопроиз- водительности ТЭСОТ. Кроме того, влияние потерь на термических сопротивлениях приводит к смещению оптимального диапазона силы тока питания ТЭМ в сторону меньших значений.

\section{Конфоликт интересов}

Автор заявляет, что у него нет конфликта интересов.

\section{Список литературы}

[1] Y.W. Chang, C.C. Chang, M.T. Ke, S.L. Chen. Appl. Thermal Eng., 29, 2731 (2009). DOI: $10.1016 /$ j.applthermaleng.2009.01.004

[2] R. Chein, G. Huang. Appl. Thermal Eng., 24, 2207 (2004). DOI: 10.1016/j.applthermaleng.2004.03.001

[3] М.Ю. Штерн, Ю.И. Штерн, А.А. Шевченков. Известия вузов. Сер. Электроника, 4, 30 (2011).

[4] А.Ф. Иоффе. Полупроводниковые термоэлементы (Издво АН СССР, М.-Л., 1960)

[5] С.В. Ордин. ФТП, 31 (10), 1269 (1997). [S.V. Ordin. Semiconductors, 31, 1091 (1997). DOI: 10.1134/1.1187031]

[6] А.В. Дмитриев, И.П. Звягин. УФН, 180 (8), 821 (2010). DOI: 10.3367/UFNr.0180.201008b.0821 [A.V. Dmitriev, I.P. Zvyagin. Physics-Uspekhi, 53 (8), 789 (2010). DOI: $10.3367 /$ UFNe.0180.201008b.0821]

[7] А.А. Шерченков, Ю.И. Штерн, Р.Е. Миронов, М.Ю. Штерн, М.С. Рогачев. Российские нанотехнологии, 10, 827 (2015).

[8] Y.X. Huang, X.D. Wang, C.H. Cheng, D.T.W. Lin. Energy, 59, 689 (2013). DOI: org/10.1016/j.energy.2013.06.069

[9] E.S. Jeong. Cryogenics, 59, 38 (2014). DOI: org/10.1016/j.cryogenics.2013.12.003

[10] Е.Н. Васильев. ЖТФ, 87 (9), 1290 (2017). DOI: 10.21883/JTF.2017.09.44899.2094 [E.N. Vasil'ev. Tech. Phys., 62 (9), 1300 (2017). DOI: org/10.1134/S1063784217090286]

[11] Электронный ресурс. Режим доступа: http://www.crystal1therm.com/upload/iblock/60b/TM-S_127_14_11_L2-SPEC.pdf

[12] Е.Н. Васильев. ЖТФ, 88 (4), 487 (2018). DOI: $10.21883 / J T F .2018 .04 .45714 .2312$ [E.N. Vasil'ev. Tech. Phys., 63 (4), 487 (2018). DOI: org/10.1134/S1063784218040266]

[13] Е.Н. Васильев. ЖТФ, 87 (1), 80 (2017). DOI: $10.21883 /$ JTF.2017.01.44022.1725 [E.N. Vasil'ev. Tech. Phys., 62 (1), 90 (2017). DOI: org/10.1134/S1063784217010248]

[14] Электронный ресурс. Режим доступа: http://kryotherm.ru/ ru/assembly-instructions.html

[15] Электронный ресурс. Режим доступа: http://ecogenthermoelectric.com/ru/texnicheskaya-podderzhka.html 